\title{
L'INFLUENCE DU DROIT PUBLIC SUR LA PROPRIETE PRIVEE IMMOBILIERE EN DROIT PRIVE TURC *
}

Prof. Dr. Turhan ESENER

§ 1. L'influence du droit public sur la notion de la propriété.

A l'instar du CCS la première partie du quatrième livre du CCT est consacrée à la propriété.

Les dispositions générales applicables à la propriété mobiliéré et à la propriété immobilière sont prévuès dans le 18 ème titre (les articles 618-631). La propriété foncière est règlémentée par le 19 ème titre (les articles 632-685).

Comme en droit suisse, la propriété n'est pas définie par le CCT. L'article 618 déclare que le propriétaire d'une chose a le droit d'en disposer librement dans le cadre de la loi. Cette règle concerne les droits du propriétaire. L'article $641 \mathrm{CCS}$, qui correspond à l'art. $618 \mathrm{CCT}$, parle des limites de la loi, alors que le texte allemand de 1 article 641 dit «Schranken der rechtsordnung».

En se basant sur cet article, la propriété est définie dans la doctrine turque (1) comme "un droit le plus large et exclusif de disposition ou de domination sur une chose dans les limites de la loin.

Il résulte de cette définition que la propriété consiste en deux éléments : A) Les droits du propriétaire, B) les devoirs du propriétaire.

* Rapport presenté au VIème Congrès International de droit Com. pare, Hambourg, 1962.

(1) Saymen - Elbir : Türk Eşya Hukuku, 1954, p. 221; Velidedeoğlu Esmer : Gayrimenkul tasarruflar, 1956, s. 185. 
A) Le premier élément de la propriété concerne les droits du propriétaire. Ce sont a) le pouvoir de disposition et b) le pouvoir d'exclusion.

a) Le pouvoir de disposition.

Selon l'article $618 \mathrm{CCT}$, le propriétaire d'une chose a le droit d'en disposer librement. En effet, le propriétaire peut employer, jouir, aliéner, grever de certaines charges, modifier, détruire la chose comme il lui plaît.

b) Le droit d'exclusion.

Selon l'alinéa 2 de l'article 618 le propriétaire peut revendiquer la chose contre quiconque la detient sans droit et repousser toute usurpation. Il s'agit là du caractère exclusif de la propriété.

B) Les devoirs du propriétaire.

Ils constituent les limites du droit de propriété. Parmi les éléments de la propriété on peut observer les devoirs que la loi impose au propriétaire. L'art. 618 déclare expressément que le pouvoir de disposition du propriétaire existe dans les limites de la loi.

Comme on le voit, le CCT (comme le CCS) n'a pas admis la conception individualiste du droit commun selon laquelle la propriété confère au propriétaire une puissance illimitée, absolue et exclusive. Au contraire, le propriétaire n'a le droit d'exercer une maitrise sur la chose que dans les limites prévues par la loi. On discute encore sur la question de savoir si le caractère illimité et absolu de la propriété est un élément intrinsèque de la propriété. On admet dans le droit moderne (2) que les restrictions légales de la propriété ne s'ajoutent pas à la propriété comme un corps étranger ou hostile, mais qu'elles naissent avec elle en faisant partie de son contenu.

Il résulte du texte de l'art. 618 qu'en principe le droit de propriété est illimité. En revanche, les restrictions impo-

(2) Tuor : Le CCS, 1942 , p. $449-450$; Homberger - Marti : FJS 290, propriété, 1942. 
sées sont de nature exceptionnelle (3). Le propriétaire a une maîtrise exclusive sur la chose aussi longtemps qu'une restriction n'est pas imposée par l'ordre juridique. Le contenu du droit de propriété se détermine selon les limites de la liberté de propriété prévues par le droit positif. Le droit de propriété illimité est semblable à la liberté de convention (eine absolute Vertragsfreiheit) (4).

En admettant les limites du droit de propriété le législateur a déjà écarté la conception individualiste. Donc en droit turc, comme en droit suisse, le droit de propriété est limité par la loi dans l'intérêt social. Le propriétaire n'a donc pas le droit d'exercer une maîtrise complète sur la chose. Dans quelle mesure peut-on limiter le droit de propriété ? La question de savoir quelle est l'ampleur de la puissance du propriétaire sur la chose, dépend de la structure économique et sociale de la société nationale et de l'organisation de l'Etat.

C'est pourquoi pour déterminer l'ampleur du droit de propriété en droit turc, il ne suffit certainement pas de consulter les lois privées. Il faut aussi rechercher les dispositions spéciales relatives au droit public. Etant donné qu'il existe une corrélation étroite entre la propriété et l'organisation de l'Etat, il est indispensable d'étudier le droit public pour déterminer les limites du droit de propriété établies dans l'intérêt social. Voici l'importance du problème de l'influence du droit public sur la propriété privée immobilière. Diverses dispositions des lois publiques concernant la propriété immobilière dovient être appréciées à la lumière de la loi constitutionnelle.

Le principe général de la propriété est énoncé par l'art. 36 de la loi constitutionnelle turque. Selon cet article "chacun a le droit de propriété. Ce droit ne peut être limité par la loi que pour des considérations d'intérêt social. L'exercise du

(3) Meiyer-Hayoz: Schweizerisches ZGB, Das Eigentum, 1959, Art. 641, No. 20.

(4) Meiyer - Hayoz: Art. 641 No. 21; cf. Oftinger : Die Vertragsfreiheit des Bürgers im Schweizerischem Recht, 1946, p. 315 et spécialement 324 . 
droit de propriété ne peut être contraire à l'intérêt de la société».

Il résulte de cet article de la loi constitutionnelle que :

a) Chaque personne jouit du droit de propriété,

b) Le droit de propriété n'est pas un droit illimité et ne confère pas à son titulaire une liberté absolue (5). Dans l'intérêt social, le législateur peut édicter des dispositions limitant le droit de propriété.

c) Le propriétaire ne peut exercer son droit de propriété contre l'intérêt de la société.

Compte tenu du principe établi dans l'article 36 de la loi constitutionnelle, il est nécessaire de démontrer l'influence des lois publiques sur la propriété immobilière.

En droit civil turc les choses immobilières (les immeubles) sont les suivantes (Art. 632 et 911):

a) Les biens - fonds.

Selon l'article 3 du réglement du registre foncier les biens fonds sont des surfaces du terrain limitées par des moyens suffisants et précis.

b) Les droits distincts et permanents immatriculés au registre foncier.

Ces droits ne sont pas, dans le sens propre du mot, un immeuble. Au fond ce sont des servitudes, comme le droit de superficie (art. 751/3) et le droit à une source sur le fonds d'autrui (Art. 752 al. 2). Ils sont simplement traités comme des choses immobilières pour des raisons économiques et techniques. Il est important à noter que le rapport entre le titulaire d'un droit distinct et permanent et le propriétaire $\mathrm{du}$ fonds grévé est régi par les dispositions concernant les servitudes et non pas par celles qui concernent les droits de voisinage.

(5) Le rapport du Commission Constitutionnelle de l'Assemblée Constituante, 5/7, Art. 36 . 
c) Les mines.

En droit turc, les mines sont régies par la loi minière No. 6309 du 1954. Selon le principe admis par le CCT, la propriété du sol comporte celle du dessus et du dessous dans toute la hauteur et la profondeur utiles à son exercice. Mais les mines sont soumises à une autre régime juridique. Selon l'art. 14 de cette loi les mines sont soumises au droit de disposition de l'Etat et non pas au droit de disposition du propriétaire du sol dans lequel elles se trouvent.

Selon l'article 9 al. 1, la recherche des mines dépend de l'obtention d'un license.

\section{§ 2. L'influence du droit public sur la propriété privée immobilière.}

Ayant ainsi relevé trés brièvement l'objet de la propriété immobilière, nous pouvons démontrer, dès lors, l'influence du droit public sur la propriété privée immobilière. Les restrictions du droit de propriété immobilière résultent en partie d'actes juridiques, qui donnent naissance à des droits rééls particuliers et en partie de la loi, c'est ce qu'on appelle les restrictions ou les limites légales de la propriété.

Comme nos recherches sont destinées à démontrer exclusivement l'influence du droit public sur la propriété immobilière privée, les biens du domaine public qui ne sont pas considérés comme objet de la propriété privée (6) et qui sont affectés à l'usage commun, tels que les eaux publiques, les regions impropres à la culture, rochers éboulis, névés, glaciers et les sources en jaillisant (Art. 641 CCT), sont laissés hors du cadre de cette étude.

Les limites légales de la propriété immobilière peuvent découler des dispositions du droit privé ou du droit public. Le but des restrictions de la propriété immobilière prévues par le droit civil est de sauvegàrder soit les intérêts particuliers des voisins (Rapport voisinage Art. $661 \mathrm{CCT}$; fouilles

(6) cf. Wieland : Art. 664, No. 1, p. 177 (le code civil suisse I, 1913, Traduction Bovay). 
et constructions Art 662, $663 \mathrm{CCT}$; la fontaine nécessaire Art. $683 \mathrm{CCT}$ ) soit les intérêts des personnes d'un cercle plus étendu (le droit d'accés sur le fonds d'autrui art. $675 \mathrm{CCT}$; le droit de reprendre les épaves ou les animaux échappés art. 676 CCT. Cas de nécessité Art 677 CCT).

En revanche, le but des restrictions prévues par le droit public est de sauvegarder l'intérêt de la société, de la collectivité. En d'autres termes, les restrictions de droit public sont instituées dans l'intérêt général.

Les restrictions de la propriété privée immobilière dérivant du droit civil et du droit public présentent une particularité commune. Selon l'article 657 du CCT les restrictions légales de la propriété sont valables sans qu'il y ait lieu de les inscrire au registre foncier (7).

Dans la doctrine (8), on a souligné que les deux catégories de restrictions légales offrent des différences importantes. Les différences en question peuvent être résumées en deux points :

1) La première différence se présente en cas de contestation ou de violation de l'exercice du droit découlant de la restriction légale. Les restrictions de la propriété immobilière résultant du droit civil confèrent à leur titulaire une véritable action devant les tribunaux civils, alors qu'une décision concernant les restrictions résultant du droit public ne peuvent être prises en considération "ex officio» que par des autorités ou tribunaux administratifs.

(7) Il est à noter toutefois que cette règle ne s'applique qu'aux restrictions légales expressément prévues par le CCT. Il y a encore d'autres restrictions que la loi ne regie pas elle-même, mais dont elle se borne à laisser l'institution a la volonté des intéréssés (Par. ex. selon l'al. 2 de l'art. 668, les installations d'une conduite sont, à la requête de l'ayant droit, inscrites à ses frais au registre foncier; le droit de passage constitué d'une manière permanente doit être inscrit au registre foncier (Art. $672 \mathrm{CCT}$ ); de même pour la fontaine nécessaire (art. $351 \mathrm{du}$ réglement du registre foncier).

(8) Tuor : p. 482; Saymen - Elhir : p. 372; Wieland : art. 380 No. 1 , p. 301 . 
2) Les restrictions de droit privé, n'étant établies que dans l'intérêt de tel ou tel particulier, peuvent être suprimées ou modifiées par un acte authentique et une inscription au registre foncier (Art. 657/2 CCT). En revanche, les restrictions établies das l'intérêt public ne peuvent être ni modifiées, ni supprimées (Art. 657/3 CCT). En effet, les restrictions provenant du droit public son de nature impératives. Les conventions de droit privé (une rénonciation, par exemple) ne sauraient porter sur elles (9).

En principe les restrictions du droit de propriété émanant du droit public, ne sont pas contenu dans le CCT. Toutefois le CCT, comme le CCS a exceptionnellement laissé aux lois spéciales le soin de déterminer quelques restrictions du droit de propriété. Dans ce cas la même prescription légale peut avoir pour but de protéger des intérêts généraux et des intérêts particuliers et de donner naissance à des restrictions de l'un et de l'autre catégories. Nous pouvons donner à cet égard les trois exemples suivants :

a) D'après l'article $663 \mathrm{CCT}$, les lois spéciales déterminent des distances que les propriétaires sont tenus d'observer dans les fouilles et constructions.

Ainsi la loi concernant l'organisation de direction de la construction de la ville d'Ankara No. 1351 (modifiée plus tard par les lois No. 1431, 1504, 1663, 2289, 3196) et la loi de la municipalité No. 1580 contiennent quelques dispositions touchant les distances à observer.

b) Selon l'article $673 / 2 \mathrm{du}$ CCT, l'obligation de clore les fonds et le mode de clôture sont déterminés par les lois spéciales.

Selon l'article 18 de la loi sur le cadastre et du registre foncier, le propriétaire d'un immeuble (se trouvant dans le plan de cadastre) dont les limites ne sont pas déterminées doit mettre des signes de démarcation conformément au coutume.

(9) Wieland: art 702, No. 6 . 
L'article 47 de la loi No. 2290 du 10 juin 1933 sur les routes et constructions municipales prévoit que les propriétaires du terrain doivent limiter leur immeuble par démarcation sur le terrain dans une année à partir de la date où le plan de la ville devient définitif.

c) Selon l'article $675 / 2$ du CCT, la mesure en laquelle il est permis de pénétrer dans le fonds d'autrui pour la chasse ou la pêche est déterminée par la loi spéciale. Par exemple l'article 23 de la loi forestière No. 3316 du 8 février 1937 dispose que «la chasse dans les forêts, le ramassage les cônes, des graines de la forêt etc, des produits du terran, la cueillette des fruits, des feuilles des arbres, la coupe des arbres secs ou les racines sont soumises à la permission de l'administration forestière. Les conditions et les durées sont également fixées par cette administration.

\section{§ 3. Les restrictions de la propriété privée immobilière résultant du droit public.}

Le CCT ne contient pas une disposition semblable à l'article 702 CCS qui dispose «Est réservé le droit de la Confédération, des cantons, et des communes d'apporter dans l'intérêt public d'autres restrictions à la propriété foncière, notamment en ce qui concerne la police sanitaire, la police des constructions, du feu, des forêts et des routes, les chemins de halage, le bornage et les signaux trigonométriques, les améliorations du sol, le morcellement des fonds, les réunions parcellaires de fonds ruraux ou de terrains à bâtir, les mesures destinées à la conservation des antiquités et des curiosités naturelles ou à la protection des sites et des sources d'eaux minérales.».

Il est souhaitable à notre avis d'inserrer une disposition pareille pour affirmer que les restrictions du droit public prévues dans les lois spéciales sont réservees. En effet, le législateur Turc a prévu plusieurs restrictions du droit de propriété immobilière dans les lois spéciales en vue de protéger l'intérêt public et social. Nous observerons qu'en droit turc le droit de propriété immobiliere est limitée au fur et à mesure par les lois du droit public. Une recherche rapide nous démontrera comment et dans quelle mesure les lois du droit 
public restreigne le droit de la propriété. Toutefois, comme les lois du droit public sont très nombreuses, il convient de s'arrêter seulement sur quelques cas caractéristiques et importants.

Les raisons légales des restrictions de la propriété immobilière sont très différentes. La plupart des restrictions tendent à limiter le droit de jouissance et le droit de disposition découlant de la propriété. Parfois, ces restrictions amènent l'extinction du droit de propriété privée immobilière.

I. - Les restrictions légales résultant du droit public tendant à restreindre le droit de jouissance du droit de la propriété immobilière.

1) Les rsetrictions résultant de la souveraineté en matière militaire.

Quelques restrictions de droit public sont prévues dans l'intérêt de la défense nationale. La loi de la zone interdite militaire datée du 27 Juin 1927, No. 1110 prévoit deux espèces de zones interdites. La première zone interdite est la zone militarisée où personne ne peut entrer, sauf les intéressés. Les immeubles se trouvant dans la première zone interdite sont assujettis à l'expropriation contre le remboursement de leur valeur (Art. 1 modifié par la loi 4944 datée du 14 juillet 1946).

2) Les restrictions légales de la propriété immobilière en raison de la nationalité étrangère de l'acquèreur.

En matière de l'exercice de droit civil, le droit privé turc admet en principe l'assimilation complète des étrangers aux nationaux. Autrement dit, l'acquisition de l'immeuble est reconrrue à chacun par le CCT.

Le principe gouvernant l'acquisition de l'immeuble par les étrangers en Turquie, est établi par l'art. 35 de la loi No. 2644 du 22 décembre 1934 sur le registre foncier.

D'après cet article les personnes physiques étrangères peuvent acquérir d'une manière réciproque les immeubles en Turquie sous la réserve des dispositions restrictives des lois. Comme on le voit, la loi soumet l'acquisition des immeubles en Turquie par les étrangers au principe de la réciprocité. 
Les auteurs Turcs (10) ont reconnu à juste titre, que la réciprocité doit être interprété dans un sens large. Par conséquent, l'expression de la réciptrocité signifie non seulement «réciprocité conventionnelle» mais aussi «réciprocité effective».

Pour observer l'influence du droit public sur la propriété immobilière, il est intéressant de déterminer les restrictions légales mentionnées par l'art. 35 de la loi sur le registre foncier.

a) L'art. 87 de la loi rurale No. 442 du 18 mars 1924 .

Selon cet article les étrangers ne pouvent acheter des immeubles dans les villages.

b) L'article 37 de la loi du registre foncier.

Selon cet article les personnes physiques étrangères ne peuvent acquérir plus de 30 hèctares du terrain hors des villages et les fermes isolées, qui ne sont pas attachées à un village que sur une permission spéciale du gouvernement. Cette règle n'est pas applicable à la succession légale.

c) L'article 1 de la loi No. 1062 du 18 mai 1927 sur les mesures de rétorsion.

Cet article prévoit comme mesure de retorsion que les immeubles d'un étranger en Turquie peuvent être saisies par le gouvernement Turc si le gouvernement de cet étranger a limité ou supprimé le droit de propriété immobilière des ressortissants Turcs par les décisions administratives ou par les lois exceptionnelles.

Le revenu résultant de la liquidation des immeubles de l'étranger peut être attribuè aux ressortissants Turcs qui ont subi des préjudices à l'étranger.

3) Les restrictions prévues pour les raisons techniques de la reconstruction.

a) Selon l'article 32 de la loi reconstruction No. $6785 \mathrm{du}$ 9.7.1956 les bâtiments à construire ne sauraient bâtir devant

(10) Par ex. Berki, Osman : Devletler hususî Hukuku, 1961, T. I, s. 202. 
la ligne déterminée par les plans de la reconstruction et de la direction des routes.

b) La loi de la circulation et de la route No. 6085 du 11 mai 1953 a également apporté quelques dispositions relative à la restriction du droit de la propriété dans l'intérêt de la sécurité routière. En effet selon l'article 12 de cette loi, il est interdit de construire les établissements tels que usine, restaurant, café, hôtel, résidence, garage, à une distance de $15 \mathrm{~m}$. sur chaque coté de la route hors de la municipalité sans obtenir un licence de la direction générale du département routière.

II. - Les restrictions légales du droit public pour des motifs d'ordre social.

1) Les interdictions de morcellement :

Le morcellement du sol présente un danger pour l'économie agricole. Le législateur suisse à cherché à parer ce danger par deux dispositions :

La première concerne le droit de succéssion (le partage, article $616 \mathrm{CCS}$ ). L'art. 616 prévoit que «les cantons ont le droit de préscrire que les biens - fonds ne pourront être morcelés au delà d'un minimum de contenance fixé pour les différentes espèces de culture». Ainsi les cantons peuvent établir des restrictions en matière de partage successoral ou bien ils peuvent interdire le morcellement d'une façon tout à fait générale (en se fondant sur l'art. $702 \mathrm{CCS}$ ) (11).

La deuxième disposition concerne la vente d'immeuble. En effet l'art. 218 al. 1 du CO déclare que le législation cantonale peut prescrire que l'acquereur d'une exploitation agricole n'aura pas le droit d'en vendre des parcelles avant l'expiration d'un délai déterminé (12).

(11) Tuor: p. 376.

(12) En droit Suisse, il existe plusieurs arrêts du Tribunal fédéral sur ce sujet. Voir Guhl : Le droit lédéral des obligations, 1947, p. $222-223$. 
Le droit turc contient ni l'une, ni l'autre de ces dispositions.

Toutefois, quelques lois de droit public contiennent aussi des dispositions relatives aux interdictions de morcellement.

a) La loi forestière.

Par exemple l'art. 71 de la loi 3116 du 8 février 1937 prévoit que les forêts privées qui donnent du bois de construction ne peuvent être ni vendues ni partagées entre les héritiers en morcelant moins de 1000 hectares et les forêts qui donnent du bois de chauffage moins de 200 hectares.

b) La loi de reconstruction No. 6785 du 9.7.1956.

L'article 37 de cette loi a prévu plusieurs règles concernant l'interdiction de morcellement selon que l'immeuble se trouve hors ou dans les limites de la municipalité.

Cette loi contient également des règles relatives à la réunion parcelaire de terrains qui peuvent être considérées commie restriction du droit de propriété.

2) Les restrictions résultant des dispositions des lois établies dans l'intérêt social et économic.

a) Distributions des terres aux fermiers.

La Turquie est essentiellement un pays agricole. Environ le $80 \%$ de la population s'occupe de l'agriculture. La productivité agricole a une importance capitale. C'est pourquoi, le législateur doit attacher une importance toute particulière aux problèmes agricoles.

L'art. 37 de la nouvelle loi constitutionnelle déclare expressement que l'Etat peut prendre les mesures nécessaires pour favoriser l'exploitation rationnelle du sol cultivable et pour procurer des terres aux fermiers qui n'en ont pas suffisamment. La loi peut indiquer la dimension des terres prenant en considération les différentes régions agricole. L'article 38 de la même loi parle de l'attribution des terres. Cela montre bien que la loi constitutionnelle impose publications des nouvelles lois qui prévoient une réforme en matière du droit d'agricole. Récemment, un projet de loi sur la réforme des terres 
a été préparé par le ministère de l'agriculture (13). Mais sur ce sujet, la tâche des juristes n'est pas encore términée et tout porte à croire que d'autres projets de loi seront préparés.

En droit Turc, la loi No. 4753 du 11 juin 1945 (modifiée par la loi No. 5618 du 22 mars 1950) sur l'attribution des terres aux fermiers est en vigueur. Cette loi tend à résoudre les problèmes sociaux relatifs à l'agriculture et à l'économie (14).

L'objectif de cette loi est ainsi défini par son premier article: «Rendre propriétaire les fermiers qui ne possèdent pas suffisamment de sol cultivable pour assurer leur subsistance ainsi que celle de leur famille et assurer aussi l'exploitation continuelle des terres».

Les terres à distribuer selon cette loi sont les suivantes : Les terres qui se trouvent à la disposition de l'Etat; les terres sans maître; les terres dérivant de drainage et d'assèchement par l'état; les terres qui se forment par l'assèchement des lacs ou par l'alluvion et remblais des fleuves; les terres résultant de l'expropriation (15). Etant donné l'objectif de cette loi, il est normale qu'elle contienne des restrictions de la propriété immobilière dans l'intérêt social.

Ces restrictions consistent plutôt à imposer les devoirs au propriétaire. Le législateur Turc admet la transformation du droit de propriété aux devoirs dans les cas où le propriétaire emploie l'immeuble dans l'intérêt commun et social (16).

Par exemple d'après l'article 54/2 de la dite loi, les terres distribués aux fermiers ne constituent pas l'objet de la poursuite et du séquestre.

L'article 56 dispose que les terres attribués par cette loi ne peuvent pas être l'objet d'un contrat de bail.

(13) Toprak Reformu Kanunu tasarısı, Tarım Bakanlı̌̆ı, 1962.

(14) Voir Velidedeoğlu - Esmer, p. 607 et suiv.

(15) Pour les arrêts de la cour de cassasion turque, voir Tepeci; Türk Kanunu Medenîsi t. II, p. 488 - 490.

(16) cf. par exemple Saymen - Elbir : p. 227. 
Selon l'article 57, le ministère de l'agriculture a le droit de demander au tribunal de reprendre la terre attribuée et modifier le registre foncier en faveur de l'Etat, lorsque le propriétaire du sol attribué l'a abandonné sans l'exploiter ou que le sol a été loué à un tiers.

b) La loi No. 5516 du 18 janvier 1950 sur les terres qui se forment par l'assèchement des marais.

Selon le premier article de cette loi, les terres résultant de l'assèchement des marais qui se trouvent hors des limites des villages et sans maître seront enregistrées au registre foncier au nom de celui qui a effectué le drainage. Mais ceux qui sont devenus ainsi propriétaire de terrain doivent, selon l'article 9 , prendre les mesures necessaires à la poursuite des opérations de drainage.

c) La loi sur l'encouragement au tourisme No. $6086 \mathrm{du}$ 22 mai 1953.

Aux termes de cette loi, les immeubles tansférés par la colléctivité aux établissements de tourisme ne peuvent pas être detournés de l'utilisation qui leur est assignée.

\section{§ 4. L'Expropriation.}

L'expropriation peut être définie comme suit: L'expropriation est un acte par lequel d'autorité publique acquiert un immeuble à titre originaire et par une décision unilatérale en se fondant sur sa souveraineté et dans l'intérêt public contre paiement préalable et entière de la valeur réélle (17).

L'expropriation est le mode de restriction de la propriété immobilière le plus important. Par cet acte, on porte atteinte aux droits acquis d'un particulier.

L'expropriation entraine l'extention du droit de propriété immobilière privée. Mais, l'acte d'expropriation est justifié par l'intérêt public. Quand il s'agit de l'intérêt public, il est permis de sacrifier en partie l'intérêt d'un individu.

(17) cf. Velidedeoğlu - Esmer : p. 514; Onar : Idare hukukunun umumî esaslan, 1960, p. 1087. 
Le principe fondamental de l'expropriation est établi par l'article 38 de la nouvelle loi constitutionnelle. Cette article déclare que «les personnes morales de droit public sont autorisées à exproprier la propriété immobilière privée contre le remboursement de la vaelur réelle, conformément à la procédure indiquée par la loi et à constituer des servitudes administratives sur ces immeubles.

L'art. 74 de l'ancienne loi constitutionnelle prévoyait le paiement d'avance de la contre valeur de la propriété expropriée. Mais le second alinéa de l'article 38 de la nouvelle loi constitutionnelle a apporté une exception importante en modifiant cette règle. La mode de paiement de la valeur des terrains expropriés en application de la loi sur l'attribution des terres aux fermiers, l'Etatisation des forêts est déterminé par la loi spéciale. Dans les cas où la loi prévoit le paiement par acompte, la durée de ce paiement ne peut excéder dix ans. Dans ce cas, le paiement est effectué par des versements égaux. Le taux d'intérêt est fixé par la loi.

En attendant la publication des nouvelles lois spéciales conformément à la nouvelle loi constitutionnelle, il est utile de jeter un coup d'oeil aux lois en vigueur relatives à l'expropriation.

A ce propos, le code civil turc continent deux dispositions : Selon l'article 633 al. 2 «celui qui acquiert un immeuble par expropriation en devient propriétaire avant l'inscription mais, il ne peut en disposer qu'après l'inscription au registre foncier».

L'article 643 al. 2 CCT dispose d'autre part qu'en cas d'expropriation pour cause d'utilité publique, le moment où la propriété s'éteint est déterminé par les lois spéciales.

Bien que l'article 643 renvoie cette question aux lois spéciale, la cour de cassassion l'a résolue en prononçant que l'acquisition de la propriété immobilière expropriée ne se produit pas au moment où la décision d'expropriation est rendue mais seulement par le paiement du prix de l'expropriation (18).

(18) Cour de cassasion, ch. 3 arrêt, No. $7785 / 5880$ du 10.5.1951, Velidedeoğlu - Esmer, p. 515, Note 2. 
En droit turc, l'expropriation est reglée par la loi d'expropriation No. 6830 du 31. Août 1956.

L'article 35 de la même loi a promulgué les dispositions du décret d'expropriation de 1869; de la loi de l'expropriation à l'égard des besoins de défense nationale No. 3887; de la loi sur l'expropriation de chemin de fer No. 929 et la loi sur l'expropriation par municipalité No. 3710 dans la mesure où elles sont incompatibles avec celles de cette loi.

Pour l'expropriation d'un immeuble privé, il faut que deux conditions soient réunies :

a) La décision d'utilité publique,

b)Le payement d'avance de la contre valeur de l'immeuble exproprié.

La décision de l'intérêt public prise par les personnes morales de droit public doit être ratifiée par l'autorité compétente. Seulement les décisions d'intérêt public prises par le Conseil des ministères ne sont pas soumises à une telle approbation.

L'autorité public peut acheter l'immeuble par un accord ¿̀ l'amiable avec le propriétaire sur le prix fixé par la commission formée à cette effet pendant la procédure d'expropriation. Dans ce cas l'acquisition de l'immeuble par expropriant est considérée, d'après l'article 8 al. 2, comme une acquisition par voie d'expropriation.

Si l'on prend en considération cette règle, vis à vis du $\mathrm{CCT}$, on arrive au résultat suivant: l'achat d'un immeuble par la commission d'expropriation sur un accord à l'amiable avec le propriétaire étant considéré que comme dans l'expropriation, l'expropriant en devient propriétaire avant l'inscription au registre foncier par application de l'art. $633 \mathrm{CCT}$.

Quant au paiement de la contre valeur de l'immeuble exproprié, la loi dispose que le prix de l'expropriation sera déterminé par une commission d'appréciation.

Le propriétaire de l'immeuble, le possesseur, d'autres intéressés ou l'expropriant lui-même peuvent contester devant un 
trubunal civil la décision de la commission d'appréciation (Art. 14). En revanche, le propriétaire de l'immeuble ne peut recourir contre l'acte d'expropriation qu'au Conseil d'Etat et non aux tribunaux civils.

L'article 17 concerne l'immatriculation au registre foncier au nom de l'expropriant. Lorsque le propriétaire n'a pas contesté le prix d'expropriation en temps utile et qu'il s'est abstenu de remplir les formalités nécessaires de l'aliénation devant l'officier du registre foncier, l'expropriant déposant le prix d'expropriation à une banque nationale, a le droit de demander l'inscription au registre foncier. Le tribunal invite immédiatement les deux parties et ordonne l'inscription en faveur de l'expropriant.

La décision de l'expropriation constitue elle-même une restriction de la propriété immobilière résultant du droit public. En effet, dès le moment de la notification de la décision au propriétaire, celui-ci n'a plus le droit de construire un nouveau bâtiment ou de procéder à des modicifations importantes.

§ 5. La restriction de la propriété immobilière privée résultant de la loi pétrolière No. 6326 du 16 mai 1954 modifiée par la loi No. 6558 et 6987.

En droit turc, les sources de pétrole, comme les mines, se trouvent en la possession de l'Etat (Art. 1).

L'article 87 ce cette loi comporte une règle importante au point de vue de la restriction de la propriété immobilière : le possesseur d'un droit pétrolier peut acquerir le droit d'utiliser le terrain nécessaire pour les opérations pétrolières par un accord à l'amiable avec le propriétaire du sol ou par voie d'expropriation.

La décision d'expropriation est rendue par l'administration pétrolière. Cétte décision est considérée comme étan: d'intérêt public. Par conséquent, la loi sur l'expropriation est applicable dans cette matière. 